\title{
UNIQUENESS OF PLANE EMBEDDINGS OF SPECIAL CURVES
}

\author{
SHREERAM S. ABHYANKAR AND AVINASH SATHAYE \\ (Communicated by Wolmer V. Vasconcelos)
}

\begin{abstract}
For a family of special affine plane curves, it is shown that their embeddings in the affine plane are unique up to automorphisms of the affine plane. Examples are also given for which the embedding is not unique. We also discuss the Lin-Zaidenberg estimate of the number of singular points of an irreducible curve in terms of its rank. Formulas concerning the rank of the curve lead to an alternate simpler version of the proof of the Epimorphism Theorem.
\end{abstract}

\section{INTRODUCTION}

Let $f=f(X, Y)$ be a nonconstant bivariate polynomial with coefficients in an algebraically closed field $k$ of characteristic zero. The following very remarkable result about the affine plane curve $f=0$ was obtained by Lin and Zaidenberg [LZ1] by using complex analysis. It was reproved by Neumann and Rudolph [NeR] by topological methods.

Lin-Zaidenberg Theorem (1.1). If $C: f(X, Y)=0$ is an irreducible affine plane curve of genus zero having only one place at infinity, such that all the singularities of $C$ are unibranch, and $C$ does have at least one singularity at finite distance, then $C$ is equivalent to $Y^{n}-X^{m}=0$ for some integers $n>1<m$ with $G C D(n, m)=1$.

We will, as usual, call the residue class ring $k[X, Y] /(f)$ the coordinate ring of the curve $C: f=0$. Recall that the curve $C$ is irreducible if its coordinate ring is an integral domain, or equivalently if its equation is irreducible. For an irreducible curve $C$, recall that the quotient field of the coordinate ring of $C$ is termed its function field.

Recall that $f$ (i.e., the curve $f=0$ ) has only one place at infinity means that it is irreducible and further that its coordinate ring is contained in all except one DVR (= discrete valuation ring) of its function field (over $k$ ). By genus of the curve $f$ we will mean the genus of its function field.

Note that then the phrase " $f$ has genus zero and only one place at infinity" is equivalent to " $f$ is irreducible in $k[X, Y]$ and $f(u(T), v(T))=0$ for some $(u(T), v(T)) \in k[T]^{2} \backslash k^{2} "$.

Received by the editors October 24, 1994.

1991 Mathematics Subject Classification. Primary 13B10, 13B25, 14C40, 14H20.

This work was partly supported by NSF grant DMS 91-01424 and NSA grant MDA 904-95$\mathrm{H}-1008$. 
Also recall that a singularity of $f$ (at finite distance) is a point $(a, b) \in k^{2}$ such that $f(a, b)=f_{X}(a, b)=f_{Y}(a, b)=0$. Moreover, this singularity is unibranch means $f(X+a, Y+b)$ is irreducible in the (formal) power series ring $k[[X, Y]]$.

Recall that $g \in k[X, Y]$ is equivalent to $f$ means $g=\tau(f)$ for some $k$ automorphism $\tau$ of $k[X, Y]$. Likewise, $g$ is said to be isomorphic to $f$ if the residue class rings $k[X, Y] /(g)$ and $k[X, Y] /(f)$ are $k$-isomorphic. Finally, $f$ has a unique embedding means every $g$ which is isomorphic to $f$ is equivalent to $f$.

Now here is an immediate corollary of the above theorem.

Corollary (1.2). If $f=Y^{n}-X^{m}$, where $n>1<m$ are integers with $G C D(n, m)$ $=1$, then $f$ has a unique embedding.

In Section 2, for the case when $n$ and $m$ are prime numbers, we shall generalize (1.2) by proving the following:

Uniqueness Theorem (1.3). If $f=Y^{n}-X^{m}+\sum_{i n+j m<m n} a_{i j} X^{i} Y^{j}$ with $a_{i j} \in$ $k$, where $n \neq m$ are prime numbers, then $f$ has only one place at infinity and $f$ has a unique embedding.

In Section 3, for the case when $n$ (or $m$ ) is not a prime number, we shall prove the following:

Nonuniqueness Theorem (1.4). If $n>1<m$ are integers with $G C D(n, m)=1$ such that $n$ is not a prime number, then it is possible to construct a curve defined by $f=Y^{n}-X^{m}+\sum_{i n+j m<m n} a_{i j} X^{i} Y^{j}$ with $a_{i j} \in k$, such that $f$ has one place at infinity but at least two (nonequivalent) embeddings.

In Section 4, we shall give a high-school version of (1.1), and in Section 5, we shall discuss the Lin-Zaidenberg conjecture generalizing it. Finally, in Section 6, we give a more detailed explanation of the conjecture. Thereby we obtain alternate simpler proofs of the Epimorphism Theorem and some of the arguments in the Lin-Zaidenberg Theorem.

\section{UNIQUENESS}

We now give a proof of the Uniqueness Theorem (1.3).

First, we need to discuss the concept of equivalence of embeddings in greater detail. Given a curve $f$ with one place at infinity, we have the degree-semigroup $\Gamma(f)$ associated with it, which is simply defined by $\Gamma(f)=\{-v(h) \mid 0 \neq h \in$ coordinate ring of $f\}$. The Expansion Techniques in [Ab2] give a standard generation of this semigroup by a $\delta$-sequence $\delta_{0}=-v(x), \delta_{1}=-v(y), \ldots, \delta_{h}$. Actually, in [Ab2] the equivalent sequence $-\delta_{i}=r_{i}$ is discussed. The theory therein further constructs a standard $g$-sequence - a sequence of approximate roots of $f$ - such that $\delta_{i}=-v\left(g_{i}\right)$. If among the first two numbers $\delta_{0}, \delta_{1}$ one divides the other, then by exchanging $X, Y$ if necessary, we may assume that $\delta_{1}=b \delta_{0}$. It is easy to see that an automorphism of the form $X^{\prime}=X, Y^{\prime}=y+c X^{b}$ will cause a reduction in $\delta_{1}$ for a unique $c \in k$. Repeating this as often as needed, we may assume that neither of $\delta_{0}, \delta_{1}$ divides the other or $y=0$. The latter degenerate case is described by setting $h=0$ and we say that $\delta_{1}$ is $-\infty$ or undefined. The situation after the reduction is described by saying that $\left(\delta_{0}, \delta_{1}\right)$ is nonprincipal, or equivalently the $\delta$-sequence is nonprincipal. 
Thus, it is clearly enough to discuss when two embeddings with nonprincipal $\delta$-sequences are equivalent. A complete answer is known by ([AbS]) which states that the equivalence class is completely determined by the GCD of $\delta_{0}, \delta_{1}$.

In particular, to show uniqueness of the embedding, it is enough to show that any embedding has the same $\delta_{0}, \delta_{1}$, up to a possible transposition.

To prove that $f$ has one place at infinity, we need the following:

Irreducibility Criterion. Assume that $n=\operatorname{deg}_{Y} f(X, Y) \not \equiv 0 \bmod \operatorname{char} k$ and $f(X, Y)$ is monic in $Y$. Then $f(X, Y)$ has one place at infinity iff there is a "test series " $u(\tau) \in k((\tau))$ such that

$$
\operatorname{ord}_{\tau} f\left(\tau^{-n}, u(\tau)\right)>s_{h}(-n, u(\tau))
$$

Moreover, given any series passing this test, there is a "root" $y(\tau)$ (usually called the Newton-Puiseux series of $f$ ) satisfying:

$$
f\left(\tau^{-n}, y(\tau)\right)=0 \text { and } \operatorname{ord}_{\tau}(y(\tau)-u(\tau))>m_{h}(-n, u(\tau)) .
$$

This is reproduced from $[\mathrm{SaS}]$ and we will follow the notation therein. A more detailed discussion of the criterion is found in [Ab2] and [Ab3].

In our case, we use the test series $u(\tau)=\tau^{-m}$. For this series, the number of characteristic terms $h=1$ and $s_{h}=s_{1}=-m n$. Evidently,

$$
\operatorname{ord}_{\tau} f\left(\tau^{-n}, u(\tau)\right)=\min \left\{-j n-i m \mid a_{i, j} \neq 0\right\}>-m n
$$

and we get that $f$ has one place at infinity. Moreover, from the last part of the criterion we deduce that the degree-semigroup of $f$ must be generated by the $\delta$ sequence $(n, m)$ (since the $g$-sequence is simply $(X, Y)$ here).

Finally, we show uniqueness. Recall that an element of a semigroup is termed primitive if it is not a sum of two nonzero elements of the semigroup. The prime numbers $n, m$ in the semigroup are clearly primitive by the topic "Primitivity of Generators" in 6.3.1 [SaS]. The topic "Prime Numbers in a Planar Semigroup" following it shows that the only possible nonprincipal $\delta$-sequences generating the degree-semigroup of $f$ are $(n, m)$ or $(m, n)$. Thus, we have uniqueness of embeddings as explained above. The proof can also be deduced by the results from [Ab2].

\section{NONUniqueness}

We give a proof of the Nonuniqueness Theorem (1.4).

Write $n=a b$ where $a, b$ are both positive integers bigger than 1 . Consider the curve defined by $g=\left(Y^{a}-X^{m}\right)^{b}-Y$. From the irreducibility criterion, it is easily seen that the curve $g=0$ has one place at infinity. (Use a test series $\tau^{-m b}\left(1+\frac{1}{a} \tau^{m(a b-1)}\right)$.)

Set $x, y, z=y^{a}-x^{m}$ to be the residue class images of $X, Y, Y^{a}-X^{m}$ respectively modulo $g$. It is easy to verify that the orders at the unique valuation $v$ at infinity must satisfy $v(x)=-a b=-n, v(y)=-m b, v(z)=-m$. Moreover, by definition, the coordinate ring of $g=0$ is generated by $x, y$. Since $y=z^{b}$ modulo $g$, the ring $k[x, y]$ is obviously contained in $k[x, z]$. Since the reverse inclusion $k[x, z] \subset k[x, y]$ 
is obvious, we get that the coordinate ring of $g$ is obviously generated by $x, z$ as well.

The minimal equation satisfied by $x, z$ is obviously $z=z^{a b}-x^{m}$. Thus the degree-semigroup is generated by two different $\delta$-sequences $(n, m b, m)$ and $(n, m)$. Since the GCD of $\delta_{0}, \delta_{1}$ is respectively $b, 1$ by the equivalence criterion in [AbS], we deduce that the embeddings are not equivalent!

\section{High-SCHOOL VERSION}

Let $(n, m)$ be the bidegree of $(u(T), v(T)) \in k[T]^{2} \backslash k^{2}$, i.e., let $n$ and $m$ be the degrees of $u(T)$ and $v(T)$ respectively. The resulting polynomial curve parametrized by $X=u(T), Y=v(T)$ has one place at infinity and hence we can arrange the bidegrees to be nonprincipal as explained above. Here it is easier to show the process explicitly thus:

Note that if $n$ or $m$ is not an integer, then $(n, m)$ is nonprincipal by convention. In view of this, since the degree of the zero polynomial is $-\infty$, we see that if either $u$ or $v$ is zero, then $(n, m)$ is nonprincipal.

If $m$ divides $n$, then we can get a reduction in the bidegree by replacing $(u, v)$ by $\left((u / U)-(v / V)^{n / m}\right)$ where $U$ and $V$ are the coefficients of $T^{n}$ and $T^{m}$ in $u$ and $v$ respectively. Repeating this a finite number of times we can arrange matters so that the bidegree is nonprincipal. Therefore (cf. [Ab1] and [AbS]) we see that (1.1) is equivalent to the following:

High-School Version of Lin-Zaidenberg Theorem (4.1). Given any $u(T)=$ $T^{n}+\sum_{i=0}^{n-1} u_{i} T^{i}$ with $u_{i} \in k$ and $v(T)=T^{m}+\sum_{j=0}^{m-1} v_{j} T^{j}$ with $v_{j} \in k$, where $1<n<m$ are integers with $m \neq \equiv 0(\bmod n)$, we have the following. $k$.

(4.1.1) If $G C D(n, m) \neq 1$, then $\left(u\left(t_{1}\right), v\left(t_{1}\right)\right)=\left(u\left(t_{2}\right), v\left(t_{2}\right)\right)$ for some $t_{1} \neq t_{2}$ in

(4.1.2) Suppose that $G C D(n, m)=1, u_{0}=u_{1}=0$ and $v_{j}=0$ for all nonnegative integers $j<m$ with $j \equiv 0(\bmod n)$. If $(u(T), v(T)) \neq\left(T^{n}, T^{m}\right)$, then $\left(u\left(t_{1}\right), v\left(t_{1}\right)\right)=\left(u\left(t_{2}\right), v\left(t_{2}\right)\right)$ for some $t_{1} \neq t_{2}$ in $k$.

\section{Conjecture}

Assuming that $f$ is irreducible, let $g$ be the genus of $f$. Let $P_{1}, \ldots, P_{s}$ be the singularities of $f$ (at finite distance) labelled so that upon letting $q_{i}$ be the number of branches at $P_{i}$ we have $q_{i}=1$ for $1 \leq i \leq s^{\prime}$ and $q_{i}>1$ for $s^{\prime}<i \leq s$. Set $q=\sum_{s^{\prime}<i \leq s}\left(q_{i}-1\right)$. Let $V_{\infty}$ be the branches of $f$ at infinity. Set $q_{\infty}=$ (card $\left.V_{\infty}\right)-1$, and recall that the rank $r$ of $f$ is defined by putting $r=2 g+q+q_{\infty}$. (This terminology is motivated by the fact that, in case $k=\mathbb{C}, r$ is the rank of the first homology group $H_{1}(f)$.) To generalize a part of (1.1), Lin and Zaidenberg [LZ2] have formulated the following :

Lin-Zaidenberg Conjecture (5.1). $s \leq 2 r+1$.

(The coefficient 2 is missing from [LZ2] due to a printing mistake which was corrected in [LZ3].)

Supporting calculations. Now we present some calculations in support of the Lin-Zaidenberg Conjecture (5.1).

Assume that $f$ is monic of degree $n>1$ in $Y$. For every branch $v$ of $f$, at finite distance or infinity, let $e(v)$ denote the ramification exponent of $v$ over $k(X)$. Let 
$v_{1}, \ldots, v_{s^{\prime}}, \ldots, v_{d^{\prime}}, \ldots, v_{d}$ be the ramified branches of $f$ at finite distance labelled so that the centers of $v_{1}, \ldots, v_{s^{\prime}}$ are at the unibranch singularities $P_{1}, \ldots, P_{s^{\prime}}$ respectively. Moreover, $v_{1}, \ldots, v_{d^{\prime}}$ are exactly all the singular branches of $f$ at finite distance.

Let

$$
\epsilon=\sum_{s^{\prime}<i \leq s}\left(q_{i}-2\right), \quad \delta=\sum_{1 \leq i \leq d}\left(e\left(v_{i}\right)-2\right) \quad \text { and } \quad \theta=d-s^{\prime} .
$$

Then clearly:

Lemma (5.2). $\epsilon, \delta, \theta$ are nonnegative integers.

We have the equations:

$$
\begin{gathered}
q=\sum_{s^{\prime}<i \leq s}\left(q_{i}-1\right)=\left(s-s^{\prime}\right)+\sum_{s^{\prime}<i \leq s}\left(q_{i}-2\right)=s-s^{\prime}+\epsilon, \\
\sum_{1 \leq i \leq d}\left[e\left(v_{i}\right)-1\right]=\sum_{1 \leq i \leq d}\left[e\left(v_{i}\right)-2\right]+d=\delta+s^{\prime}+\theta
\end{gathered}
$$

and

$$
\sum_{v \in V_{\infty}}[e(v)-1]=n-q_{\infty}-1
$$

where $V_{\infty}$ denotes the set of branches at infinity.

By Riemann-Hurwitz we have

$$
2 g=2-2 n+\sum_{1 \leq i \leq d}\left[e\left(v_{i}\right)-1\right]+\sum_{v \in V_{\infty}}[e(v)-1]=1-n+\delta+s^{\prime}+\theta-q_{\infty},
$$

and hence by adding $q+q_{\infty}+n-1$ to both sides we get:

Lemma (5.3). $r+n-1=s+\epsilon+\delta+\theta$.

By (5.2) and (5.3) we get:

Lemma (5.4). If $n \leq r+2$, then (5.1) is correct.

By (5.4) we see that:

Proposition (5.5). If $n \leq 4$ and $g \neq 0$, then (5.1) is correct.

By (1.1) and (5.4) we see that:

Proposition (5.6). If $n \leq 3$, then (5.1) is correct.

Here is an obvious lemma.

Lemma (5.7). We consider the usual hyperelliptic curve. Thus, let $f=Y^{2}-$ $\prod_{i=1}^{h}\left(Y-\alpha_{i}\right)^{e_{i}}$, where $\alpha_{1}, \ldots, \alpha_{h}$ are pairwise distinct elements in $k$ and $e_{1}, \ldots, e_{h}$ are positive integers. Further assume that the factors are arranged so that the $e_{1}, \ldots, e_{\mu}$ are odd integers bigger than $1, e_{\mu+1}, \ldots, e_{\nu}$ are all equal to 1 and $e_{\nu+1}, \ldots, e_{h}$ are all even integers.

Also, assume that $\sum_{i=1}^{h} e_{i}$ is an odd integer (this is equivalent to $\nu$ being odd).

Then by standard calculations, we see that $f$ has only one place at infinity, its genus equals $(\nu-1) / 2$, its number of unibranch singularities equals $\mu$, and its number of nonunibranch singularities equals $h-\nu$. Thus the value of $r$ is $h+\mu-1$ and the number $s$ of finite singular points is $h$.

The above lemma shows that 
Proposition (5.8). If $f=Y^{n}-p(X)$ with $p(X) \in k[X]$ of odd degree, then $s \leq r+1$. Moreover this bound cannot be improved.

Remark (5.9). It is natural to ask if (5.1) can be strengthened to $s \leq r+1$ at least when $f$ has only one place at infinity. However, the following example shows the answer to be negative.

Consider the curve parametrized by $x=t^{3}-3 t, y=t^{4}-2 t^{2}$. The equation $f$ comes out to be $x^{4}-y^{3}+6 x^{2} y-2 x^{2}+6 y^{2}-9 y$. The curve has one place at infinity and must have 3 singularities at finite distance. Two of them are unibranch corresponding to $t= \pm 1$, and the third is a node corresponding to $t= \pm \sqrt{3}$. Thus $r=1$ and $s=3$, showing the need for the factor 2 in the conjecture. This example is a variant of one due to Lin with two places (valuations) at infinity.

\section{ANother interpretation of the RANK}

We shall assume the notation introduced at the beginning of Section 5. We will write $r(f)$ for $r$ to emphasize its dependence on $f$. In the beginning, we need not assume $f$ to be irreducible, but we need to insure that the partial derivatives $f_{x}$ and $f_{y}$ do not have a common factor, or, in other words $f$ has only a finite number of critical points.

It is possible to argue that by a general linear change of coordinates, we may arrange $f_{y}$ to be irreducible. For technical convenience, we will also arrange $f$ to be monic in $y$. Consider the plane curve defined by $f_{y}$ and consider the divisor induced by the rational function $f_{x} / f$ on $f_{y}$. Note that the derivation $\frac{d}{d x}$ modulo $f_{y}$ when applied to $f$ gives $f_{x}$. So the value of $f_{x} / f$ at any valuation $v$ can also be found to be the difference $v(d f)-v(d x)-v(f)$.

We calculate the degree of its various components as follows.

First, the sum of the degrees at finite points $P$ outside the curve defined by $f$ coincides with the degree of the intersection cycle of $f_{x}$ and $f_{y}$ in the affine plane outside the curve $f$ and we denote it by $J^{*}(f)$.

Next, we list all the valuations of $f_{y}$ at infinity as $w_{1}, \ldots, w_{d}$ and consider three different sets $V_{-}, V_{+}, V_{0}$, defined as those $w_{i}$ where $w_{i}(f)$ is respectively negative, positive, zero. Note that due to the monicness of $f$ (and hence $f_{y}$ ), we have $w_{i}(x)<$ 0 for all $i$. It is easy to calculate the value $w_{i}\left(f_{x} / f\right)$ by $w_{i}(d f)-w_{i}(d x)-w_{i}(f)$. Let $t_{i}$ be a uniformizing parameter at $w_{i}$.

For $w_{i} \in V_{+} \cup V_{-}$it is clear that we get $w_{i}(f)-w_{i}\left(t_{i}\right)-\left(w_{i}(x)-w_{i}\left(t_{i}\right)\right)-w_{i}(f)=$ $-w_{i}(x)$.

On the other hand, for $w_{i} \in V_{0}$ we get $w_{i}(d f)+w_{i}\left(t_{i}\right)-w_{i}(x)$. If $c_{i} \in k$ is the unique element so that $w_{i}\left(f+c_{i}\right)>0$, then it is easy to see that $w_{i}\left(f+c_{i}\right)=$ $w_{i}(d f)+w_{i}\left(t_{i}\right)$.

Adding up, we get the contribution at infinity to be

$$
-\sum_{1}^{d} w_{i}(x)+\sum_{w_{i} \in V_{0}} w_{i}\left(f+c_{i}\right) .
$$

Denoting the second sum by $e(f)$, we get the total contribution to be $n-1+e(f)$.

To interpret $e(f)$, we next prove: 
Lemma (6.1). Assume that $f$ and hence $f_{y}$ are monic in $y$, and write

$$
\operatorname{Res}_{y}\left(f+\lambda, f_{y}\right)=\phi(\lambda, x)=P_{0}(\lambda) x^{N}+\ldots \text { lower terms. }
$$

For each root $a_{j}$ of $P_{0}(\lambda)$ let $N_{j}=N-\operatorname{deg}_{x}\left(\phi\left(a_{j}, x\right)\right)$. Then

$$
e(f)=\sum_{\left\{a_{j}=\text { some } c_{i}\right\}} N_{j} .
$$

For proof we note that a general translate $f+c$ of $f$ has no zeros at infinity, and hence the degree of its polar divisor coincides with $N$. For a special translate, the degree of the finite intersection of $f_{y}$ and $f+c$ drops by exactly the amount of zeros picked up at infinity, so for each $a_{j}$ we get the total drop $N_{j}$ equal to

$$
\sum_{\left\{i \mid c_{i}=a_{j}\right\}} w_{i}\left(f+c_{i}\right),
$$

hence the result.

A polynomial $f$ is said to be good at infinity if $e(f+c)=0$ for a general $c$. Any curve $f$ with one place (valuation) at infinity is easily seen to be good at infinity, since the $x$-degree of the discriminant $\operatorname{Res}_{y}\left(f+\lambda, f_{y}\right)$ is independent of the value of $\lambda$. This can be looked up in most of the treatments of the Epimorphism Theorem; specifically, we cite ([Ab1], Chapter 7 or [Ab2], Chapter III). Indeed, its calculation is built into all inductive steps of the proof. Thus, the polynomial $P_{0}(\lambda)$ must reduce to a nonzero constant, without any roots. It follows that the set $V_{0}$ must be empty, leading to $e(f)=0$.

Note that, in either case $e(f) \geq 0$.

Next, we calculate the contribution from finite points lying on the curve $f$. At any such point $P$ let $x_{P}=x+b_{1}$, where $b_{1} \in k$ is the unique choice for which $x_{P}$ vanishes at $P$. Similarly, we choose the other local parameter $y_{P}=y+b_{2}$ with $b_{2} \in k$. Let $f_{1}\left(x_{P}, y_{P}\right)=f(x, y)$ be the local expansion of $f$ at $P$.

Let the valuations of $f_{y}$ centered at $P$ form the set $V_{P}$. For any valuation $u \in V_{P}$, we clearly have $u(f)>0$ by assumption and so by a calculation similar to the one above, we get

$$
u\left(f_{x} / f\right)=u(d f)-u(d x)-u(f)=-u\left(t_{P}\right)-\left(u\left(x_{p}\right)-u\left(t_{P}\right)\right)=-u\left(x_{P}\right) .
$$

Adding up over all $u \in V_{P}$ we get the contribution at $P$ to be $-\left\langle f_{y}, x_{P}\right\rangle$, where $\left\langle f_{y}, x_{P}\right\rangle$ denotes the local intersection multiplicity of $f_{y}, x_{P}$ at $P$. Now we calculate the intersection multiplicity as the order of $f_{y}$ modulo $x_{P}$. Thus $\left\langle f_{y}, x_{P}\right\rangle$ is clearly equal to $\operatorname{ord}_{y_{P}}\left(f_{1 y}\left(0, y_{P}\right)\right)$, which is clearly equal to $\operatorname{ord}_{y_{P}}\left(f_{1}\left(0, y_{P}\right)\right)-1=\left\langle f, x_{P}\right\rangle-$ 1 .

Thus, the contribution at the point $P$ comes out to be $1-\left\langle f, x_{P}\right\rangle_{P}$. Using the fact that the degree of the divisor of $f_{x} / f$ must be zero, we get

$$
J^{*}(f)+(n-1+e(f))+\sum_{P}\left(1-\left\langle f, x_{P}\right\rangle\right)=0,
$$

where, as explained above, the sum is over all points $P$ on the curve $f$. We rewrite this as:

$$
J^{*}(f)=1-n+e(f)+\sum_{P}\left(\left\langle f, x_{P}\right\rangle-1\right) .
$$


Finally, to avoid the local translations $x_{P}$, we note that, if $q_{P}$ denotes the number of valuations of $f$ at $P$, then $\left\langle f, x_{P}\right\rangle_{P}=\langle f, d x\rangle_{P}+q_{P}$, where, as usual, $\langle f, d x\rangle_{P}$ denotes the sum of the orders of the differential $d x$ at various valuations of $f$ centered at $P$.

Thus the summation in our formula can be rewritten as

$$
\langle f, d x\rangle_{\text {finite }}+\sum q_{P}-1=\langle f, d x\rangle_{\text {finite }}+q .
$$

Here, by $\langle f, d x\rangle_{\text {finite }}$ we denote the total degree of the differential $d x$ on $f$ at finite distance.

At this point, we need to assume that $f$ is irreducible, since we want to use the genus formula.

Now we use the fact that the total degree of the divisor of any nonzero differential is $2 g-2$. Moreover, the contribution of the differential $d x$ to $\langle f, d x\rangle_{\text {infinity }}$ is easily seen to be $-n-q_{\infty}-1$. So, we get that

$$
\langle f, d x\rangle_{\text {finite }}=2 g-2+n+q_{\infty}+1
$$

Combining, we get:

$$
J^{*}(f)=1-n-e(f)+q+2 g-2+n+q_{\infty}+1=r(f)-e(f) .
$$

Thus the number $r=e(f)+J^{*}(f)$ measures the number of critical points of $f$ outside it, including the special contribution from infinity. We remark that this formula gives a simple algebraic proof of the initial topological arguments in the two proofs of the Lin-Zaidenberg Theorem (1.1) ([LZ1], [NeR]). The original arguments were based on index theorems for Milnor numbers in terms of Euler characteristics.

We also remark that this calculation leads to an alternate explanation of the original Epimorphism Theorem. Recall that the Epimorphism Theorem in characteristic 0 states that: if a plane curve $f$ is isomorphic to a line, then by an automorphism of the plane, the equation $f$ can be reduced to $y$. To see this, assume that $f$ is isomorphic to a line (or that its coordinate ring is isomorphic to $k[t])$. We remark that it is enough to deduce that for any $\lambda \neq 0$, the curve $f+\lambda$ is isomorphic to a line.

Now the value of $r(f)$ is 0 since the curve $f$ is obviously a nonsingular curve of genus zero with one place (valuation) at infinity. Thus, we get that $J^{*}(f)=0$. Also, by nonsingularity of $f$, the contribution of $\left\langle f_{x}, f_{y}\right\rangle_{P}=0$ for all points on $f$ also. Thus, evidently, $\left\langle f_{x}, f_{y}\right\rangle_{P}=0$ at all $P$ in the plane. Consequently, for any $\lambda$, the $J^{*}(f+\lambda)$ evaluates to 0 also. As is well known from the Expansion Techniques, $f+\lambda$ has one place at infinity, and so $e(f+\lambda)$ is 0 for all $\lambda$. Thus the value of $r(f+\lambda)$ is also 0 . We further know that $f+\lambda$ is nonsingular since otherwise $J^{*}(f)$ would be nonzero. Thus, $f+\lambda$ must have genus 0 and is nonsingular with one place at infinity, hence is isomorphic to a line.

\section{REFERENCES}

[Ab1] S. S. Abhyankar, On the semigroup of a meromorphic curve (Part I), Proceedings of the International (Kyoto) Symposium on Algebraic Geometry (1977), 249-414. MR 83h:14020

[Ab2] S. S. Abhyankar, Expansion Techniques in Algebraic Geometry, Tata Institute of Fundamental Research, 1977. MR 80m:14016 
[Ab3] S. S. Abhyankar, Irreducibility criterion for germs of analytic functions of two complex variables, Advances in Mathematics 74(2) (1989), 100-257. MR 90h:32018

[AbS] S. S. Abhyankar and B. Singh, Embeddings of certain curves in the affine plane, Amer. Jour. Math. 100 (1978), 99-175. MR 58:16663

[LZ1] V. Lin and M. Zaidenberg, An irreducible simply connected algebraic curve in $\mathbb{C}^{2}$ is equivalent to a quasihomogeneous curve, Dokl. Akad. Nauk SSSR = Soviet Math Dokl. $\mathbf{2 7 1}=\mathbf{2 8}$ (1983), 1048-1052 = 200-204. MR 85i:14018

[LZ2] V. Lin and M. Zaidenberg, On the number of singular points of a plane affine algebraic curve, Springer Lecture Notes in Mathematics 1043 (1984), 662-63.

[LZ3] V. Lin and M. Zaidenberg, On the number of singular points of a plane affine algebraic curve, Springer Lecture Notes in Mathematics 1574 (1994), 479.

[NeR] W. Neumann and L. Rudolph, Unfoldings in knot theory (and Corrigendum), Math. Ann. 278 and 282 (1987 and 1988), 409-439 and 349-351. MR 89j:57017b

[SaS] A. Sathaye and J. Stenerson, Plane Polynomial Curves, Algebraic Geometry and Applications (1994), 121-142. MR 95a:14032

Department of Mathematics, Purdue University, West Lafayette, Indiana 47907

E-mail address: ram@cs.purdue.edu

Department of Mathematics, University of Kentucky, Lexington, Kentucky 40506

E-mail address: sohum@math.uky.edu 